\title{
A Review on the Effectivity of the Current COVID-19 Drugs and Vaccines: Are They Really Working Against the Severe Acute Respiratory Syndrome Coronavirus 2 (SARS-CoV-2) Variants?
}

\author{
Rashed Noor ${ }^{1}$ (D) \\ Accepted: 18 June 2021 / Published online: 3 July 2021 \\ (C) The Author(s), under exclusive licence to Springer Nature Switzerland AG 2021
}

\begin{abstract}
Purpose of Review In order to eradicate the COVID-19 pandemic, scientists around the world have been working very hard for a year or more with the motto of designing effective drugs and vaccines against the severe acute respiratory coronavirus 2 (SARS$\mathrm{CoV}-2$ ). Along with the positive results with the antiviral drugs and a few commercialized vaccines, the unresponsiveness as well as some side effects of such therapies have also been noticed, possibly due to the emergence of the SARS-CoV-2 variants. Therefore, current review summarized the actual effectiveness of the antivirals and vaccines which are in current use for the treatment of the COVID-19 patients.

Recent Findings So far, some drugs have been found with hopeful results among which remdesivir and arbidol are with momentous clinical progress. Besides drug designing, vaccine development has been a major effort whereby the mRNA-1273 (Moderna) and BNT162b2 (Pfizer-BioNTech) vaccines showed the required efficacy and have been approved by the US Food and Drug Administration (USFDA).

Summary While a number of existing/repurposed/repositioned or new drugs and the currently used commercial vaccines against SARS-CoV-2 apparently seem to be effective against COVID-19 mitigation, the new variants of the virus as well as the recently increased cases raised the doubt about the usefulness of these agents. Current review figured out the efficacy of different drugs and vaccines in terms of their action potential against SARS-CoV-2 and further recommended some useful measures which may be useful for future remedies.
\end{abstract}

Keywords Antiviral drugs $\cdot$ Vaccines $\cdot$ COVID-19 pandemic $\cdot$ SARS-CoV-2

\section{Introduction}

Shattering of public health in a timely manner especially by the respiratory viral infections has a long history over 100 years starting from the Russian influenza (1889-1892), the Spanish flu pandemic (1918-1920), the Asian Influenza (1957-1959), Hong Kong Influenza in 1968, the severe acute respiratory syndrome coronavirus (SARS-CoV) epidemic (2002-2003), the Middle East respiratory syndrome

This article is part of the Topical Collection on Virology

Rashed Noor

rashednoor@iub.edu.bd

1 Department of Life Sciences (DLS), School of Environment and Life Sciences (SELS), Independent University, Bangladesh (IUB), Plot 16, Block B, Aftabuddin Ahmed Road, Bashundhara, Dhaka 1229, Bangladesh coronavirus (MERS) endemic, and the running coronavirus disease (COVID-19) pandemic since the end of December, 2019 [1, 2]. Lots of reports regarding the immunogenetics and pathogenesis of all three types of coronaviruses are available from which the dreadfulness of the SARS-CoV-2 has been principally projected through their long spike (S) protein as well as the immune evasion strategy and the variant strains which could be threatening towards the usage of current vaccines as well as the repurposed antiviral drugs and immunomodulatory agents [3-7]. So far, the dreadfulness of the SARS-CoV-2 has been noticed through the world-wide $3,698,621$ deaths of people with 171,782,908 affected cases [8]. The immune response against SARS-CoV-2 infection of the angiotensin-converting enzyme 2 (ACE2) expressing the alveolar epithelial type 2 cells along the lungs mainly involves (1) the viral escape of the host interferons (IFNs that are responsible for creating the antiviral state), thereby facilitating the uncontrolled viral replication $[3,5]$; (2) recognition of viral 
RNAs by the toll-like receptors (TLRs)-3, 7/8 $(7 / 8,3)$ followed by turning off the IFN production (although the $\mathrm{T}$ cells get activated) [3••]; (3) turning on the intracellular inflammatory pathway to generate inflammatory molecules [3]; (4) recruitment of neutrophils, monocytes, macrophages, and dendritic cells (DCs) to the site of infection creating the state of cytokine storm, i.e., the level of interleukin (IL)-6, IL-8, and IL-12 and the tumor necrosis factor- $\alpha$ (TNF- $\alpha$ ) increases $[3,4,6,7]$; (5) increased level of chemokines like CXCL10, CCL2, CCL3, and CCL5) [3••]; (6) processing of viral antigens (after entry) by the antigen presenting cells (APCs), especially by the DCs and macrophages through MHC for T cell activation (MHC and the T cell receptor, TCR interaction) followed by the immunoglobulin $\mathrm{M}$ (IgM), IgG, and IgA production by B cells $[2,3]$; (7) presentation of the viral antigens by the DCs to the $\mathrm{CD} 4+\mathrm{T}$ cells instigating differentiation into memory $\mathrm{T}$ helper 1 (Th1), Th17, and the memory T follicular helper (TFH) effector cells [3・•]; and (8) presentation of viral antigens by the virus-infected epithelial cells to the $\mathrm{CD} 8+\mathrm{T}$ cells which results in cytotoxicity together with the natural killers (NK) cells (ending in apoptosis) (Figure 1).

Among the antivirals, camostat mesylate, favipiravir, combination of lopinavir plus ritonavir, remdesivir, ribavirin, IDX-184 derivatives, Saikosaponin, arbidol, hexamethylene amiloride, and N220 peptide have been widely reported acting against SARS-CoV-2 infection, while several immunomodulatory agents such as glucocorticoids, IL-6 antagonist, fedratinib, combination of baricitinib plus fedratinib plus ruxolitinib, cepharanthine plus selamectin plus mefloquine hydrochloride, dexamethasone, Lianhuaqingwen (acting both as immunomodulatory agent and the antiviral), and Lianhuaqingwen (acting both as immunomodulatory agent and the antiviral) have also been reported to mitigate the serious COVID-19 infection [6, 7]. Besides the repurposed drugs, several vaccines are under trial with the administration stage worldwide of which mRNA-1273 (Moderna), BNT162b2 (BioNTech/Pfizer), ChAdOx1/AZD1222 (Oxford/ AstraZeneca), JNJ-78436735/Ad26.COV2.S (Johnson \& Johnson), Sputnik/Gam-Covid-Vac (Gamaleya), NVXCoV2373 (Novavax), BBIBP-CorV (Sinopharm), CoronaVac (SinoVac), and BB152/Covaxin (Bharat Biotech) vaccines are worth to note according to the registered trials as well as their effectiveness [2, 9-17].

Most vaccines are expected to impart protection against the SARS-CoV-2 variants (especially in the UK and in South Africa, known as K417N/T, E484K, and N501Y mutations within the receptor-binding domain) based on their capacity to elicit a fairly broad humoral and cell-mediated immune response [18]. However, the minor mutations in SARS-CoV-2 are not even unlikely to render these vaccines unsuccessful against COVID-19 mitigation if the variants get capable to escape the host immunity $[18,19]$. Therefore, the small chance that conceivably may make the approved vaccines less effective against the variants should be in kept in

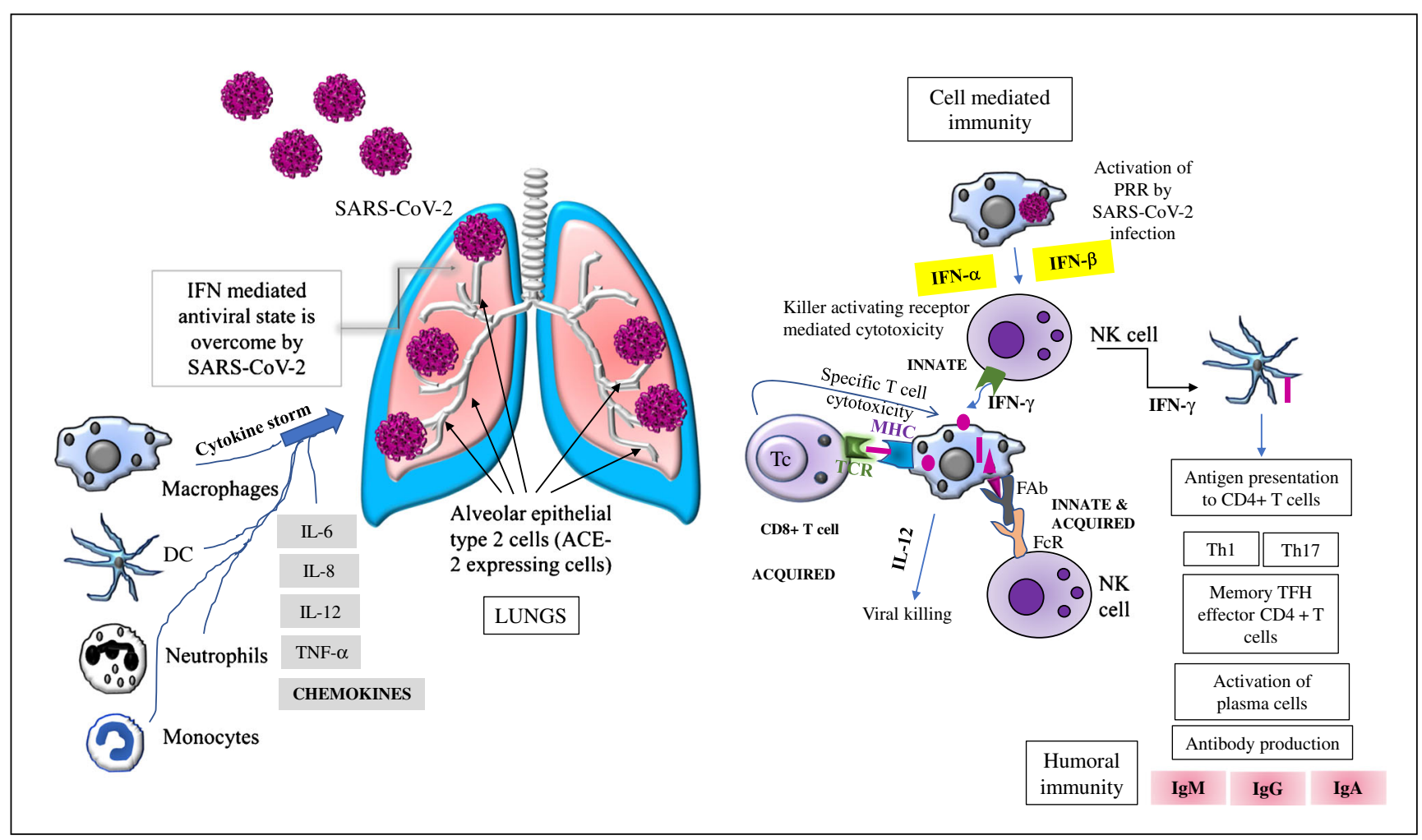

Figure 1 SAR-CoV-2 infection and host immune responses. The network between the innate and adaptive immunity as well as the humoral- and cellmediated immunity upon SARS-CoV-2 infection has been shown. Details are given in the text. 
consideration, and hence the required changes in the antigenic composition of the vaccines may be necessary. As reported earlier, the possible inactiveness of the COVID-19 vaccines due to the strain variations may demand new formulation or even the seasonal vaccination [2]. Along these lines, the current review attempted to discuss the pros and cons of the approved drugs and vaccines which are currently applied to alleviate COVID-19.

\section{Major Drugs and Their Effectiveness Against SARS-CoV-2}

In quest of effective drugs for the treatment of the COVID-19 patients, determining the efficacy of the antivirals included the in silico drug design and human studies, case-control reports and clinical trials (including the randomized controlled trials), clinical improvement, and the frequency of mortality, prospective/retrospective cohort designs, drug dosage and side effects, etc. [6, 20]. So far remdesivir, lopinavir, ritonavir, ribavirin, arbidol, ostalmovir, and favipiravir have been found to be effective, among which only remdesivir is the authorized drug approved by the US Food and Drugs Administration (FDA) for the emergency use [6, 7, 20,21]. This is to be noted that ribavirin was the most widely used antiviral for the treatment of SARS and MERS patients; however, occurrence of hemolysis and bradycardia accompanied with the elevation of the alanine transferase (ALT) level and hepatic toxicity in huge numbers of patients upon usage of this drug has been reported as well [20,21]. The administration of the antiviral lopinavir as well as the combination of lopinavir and ritonavir has been reported to elicit adversarial gastrointestinal impacts, raise of ALT level, hypokalemia, etc. [20]. Nearly similar side effects have been reported upon usage of favipiravir and arbidol too [20, 22].

\section{Generation of SARS-CoV-2 Variants}

In order to understand the genetic variability among the SARS-CoV-2, first it is worth to note that the when the virus infects host cells, the spike (S) glycoprotein which is composed of the receptor-binding subunit S1 (whose receptorbinding domain, RBD specifically interacts with the host surface receptors facilitating the viral attachment) and the membrane fusion subunit S2 (fuses host and viral membranes, releasing the viral genome into host cells) recognizes and binds to ACE2 (is the cellular receptor of SARS-CoV-2) $[2-4,6,23]$. The RBD plays a key factor of virus-receptor interactions, the host ranges, and the degree of infectivity (Figure 2) [23]. Studies on the extent of molecular differences among SARS-CoV-2 and other related coronaviruses unraveled that the SARS-CoV-2 can mutate which in turn results in the several SARS-CoV-2 variants during the ongoing pandemic as well as causing the irregular and complicated impacts on COVID-19 transmissibility and severity [23]. Since December 2019, so far more than 245,000 SARS$\mathrm{CoV}-2$ genomic sequences have been analyzed (based on the differences in two single nucleotide polymorphisms within the open reading frames ORF1ab and ORF8) which revealed the presence of only L type and S type (evolutionary older and less aggressive) variants among which $L$ type was more prevalent than $S$ type [24, 25]. However, further analysis (based on the amino acid changes) pondered the presence of A, B, C, V, G, GR, GH, T, and O variants [24-26]. As an example, the shifting from the original D614 form to the G614 variant (due to D614G mutation which replaces glycine in the spike protein) has been recently reported which influences the SARSCoV-2 binding affinity to ACE2 receptor $[24,27,28]$. Another example can be drawn which is a tri-nucleotide mutation (G28881A, G2882A, and G28883C) imparting two protein-level missense mutations, generating a cluster of over 300 variants [23].

How Do the SARS-CoV-2 Variants Hinder the Actions of Drugs?

As reported earlier, among the viral structural proteins, the most important drug target appears to be the ACE2 receptor (fused to an immunoglobulin $\mathrm{Fc}$ domain) so that the mutation within the ACE2 receptor is very much likely to inhibit the actions of saikosaponin (which binds to the spike glycoproteins and blocks the viral entry) and, arbidol, which is known to interfere with the membrane fusion, thereby restricting viral entry in to the host cells [6]. Moreover, the anti-ACE-2 antibody is known to block the viral entry and replication in Vero E6 cells since ACE2-Fc has the capacity to neutralize antibody which may further inactivate the virus $[6,29,30]$. Thus, the mutations within the ACE2 receptor discontinue the viral neutralization by the specific antiviral drugs. The occurrence of the intronic mutations (rs2106809 and rs2285666) may instigate the expression and regulation of ACE2 [31]. Besides, the evolutionary genetic linkages of a number of ACE2 polymorphisms have been reported to be linked with the pathogenesis of numerous non-communicable diseases including the cardiovascular disease (CVDs), hypertension, and diabetes mellitus [31]. Within the human ACE2 (hACE-2), amino acid sites 30-41, 82-84, and 353-357 are involved in the interaction with the SARS-CoV-2 spike (S) protein whereby the amino acid residues at the positions of $31,35,38,82$, and 353 are of significance as they are variable; and the effect of ACE2 variation due to the mutations/variation of these residues has been extrapolated towards the susceptibility to the SARS-CoV-2 [32]. Such evidences have been brought up through the animal studies like the variation at the M82N position in rat $\mathrm{ACE} 2, \mathrm{M} 82 \mathrm{~S}$, and $\mathrm{K} 353 \mathrm{H}$ mutation in mouse ACE2 and the Y217N mutation in monkey (causing ACE2 expression to be decreased and thereby reducing the viral entry) [32]. Thus, the changes in amino acid residues may 
Figure 2 The ACE-2 and RBD interaction during the initiation of SAR-CoV-2 infection. The associated drugs are shown. Details are given in the text.

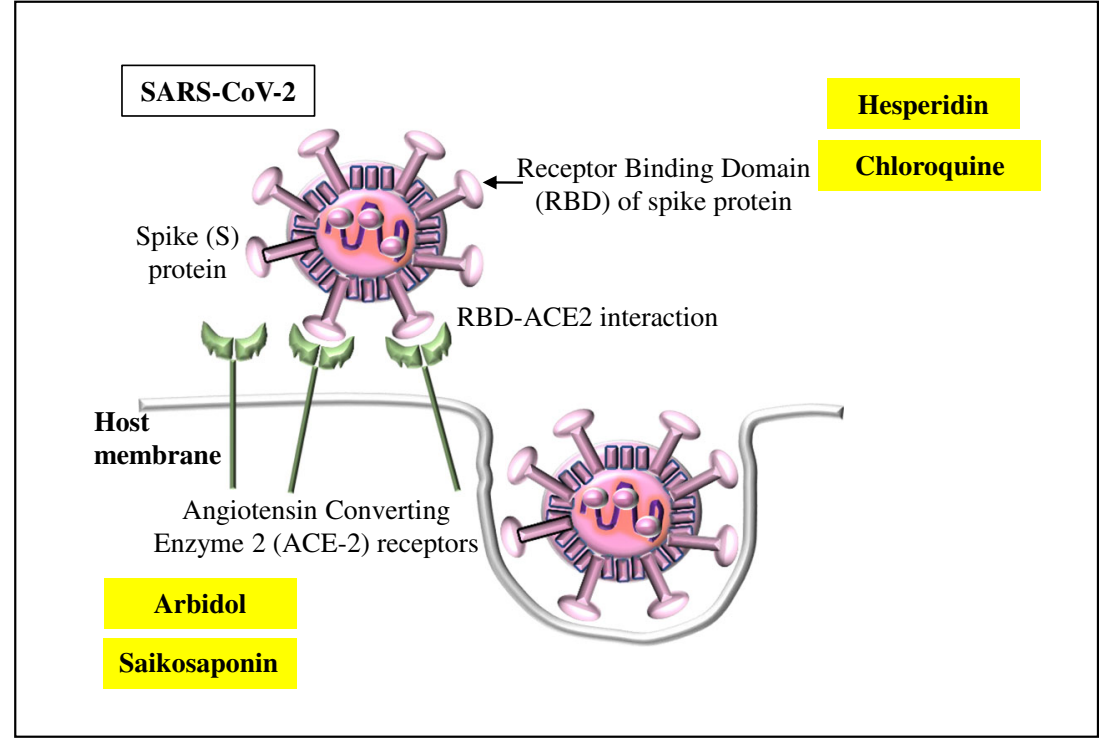

result in the lower affinity towards the viral binding; and such generic variation in the ACE2 gene can impact the susceptibility towards coronaviruses [32, 33].

Along with ACE2, the RBD is also well and widely known to be the prime target for other drugs $[6,29]$. The S1-RBD: ACE-2 interaction is usually blocked by the antiviral hesperidin resulting in the restriction of viral entry [6]. Hence the mutation within the RBD sites is likely to cancel the action of hesperidin and chloroquine [10]. As stated elsewhere, the interaction between the RBD of the spike $(\mathrm{S})$ protein and the hACE-2 augments the viral fusion into the host [34]. The positions of the amino acid residues within the $\mathrm{S}$ protein are also important since their mutations may lead to the generation of SARS-CoV-2 variants [34, 35]. Within the S protein, the extracellular N-terminus domain, the S1 subunit, the S2 subunit, the fusion peptide (FP), the heptapeptide repeat sequence 1 (HR1), the HR2 sequence, a transmembrane (TM) domain spanning across the viral membrane, and an intracellular C-terminal domain have been studied well [27, 36]. Indeed, the RBD within the S1 subunit binds to the hACE2 receptor; and FP mediates the anchoring of the target membrane $[27,35,36]$. Thus, the $\mathrm{S} 1$ and FP sites are of great interest for the study of the drug/vaccine development as they serve as potential targets. Besides, the HR region is also important for the research on therapeutic drugs against SARSCoV-2 [35, 36].

\section{Major COVID-19 Vaccines}

The research and designing/development of COVID-19 vaccines is going on with a very high speed; and the entire vaccine development process including the required clinical trials has been amazingly shortened to 15-18 months instead of 10-
15 years $[2,17]$. As a result, simultaneous marketing of several vaccines has been started from the beginning of 2021 [2, 17]. So far approximately 164 candidate vaccines are in the process of development of which 24 have been brought in the advanced stages of vaccine development [17•]. Those vaccines against SARS-CoV-2 are currently under clinical trials, whereas some of them are being administered into the target population worldwide which have been derived from any of the platforms of the inactivated/live attenuated viruses (ClinicalTrials.gov identifier NCT04412538 and NCT04324606), virus coated or protein subunit vaccines (representative trial NCT04405908), replicating/nonreplicating viral vectors (NCT04341389), DNA vaccines (NCT04368988), RNA vaccines (NCT04405076, NCT04368728), and others with the major objective to provoke the elicitation of antibodies which neutralize the spike protein of the virus $[2,9]$.

\section{Major COVID-19 Vaccines Commercially Being Used}

Among the major vaccines that are currently administered among the population worldwide, the mRNA vaccines, i.e., Pfizer-BioNTech (BNT162b1) and Moderna (mRNA 1273) vaccines, are under phase III trial with the observable efficiency $(94 \%)$ [2, 10, 11]. As reported earlier, the mRNA encoding the spike $(\mathrm{S})$ protein is protected by a submersible lipid nanoparticle; and once administered into the host, the expression of the spike protein occurs as a result of the host immune response as shown in Figure 3 [2]. The prophylactic DNA vaccine, INO-4800 is now under the phase I clinical trial (NCT04336410), has been prepared on the basis of the codon-optimized spike protein sequence to which an IgE leader sequence is attached, and the digested DNA is included into the expression plasmid pGX0001 resulting in the production 

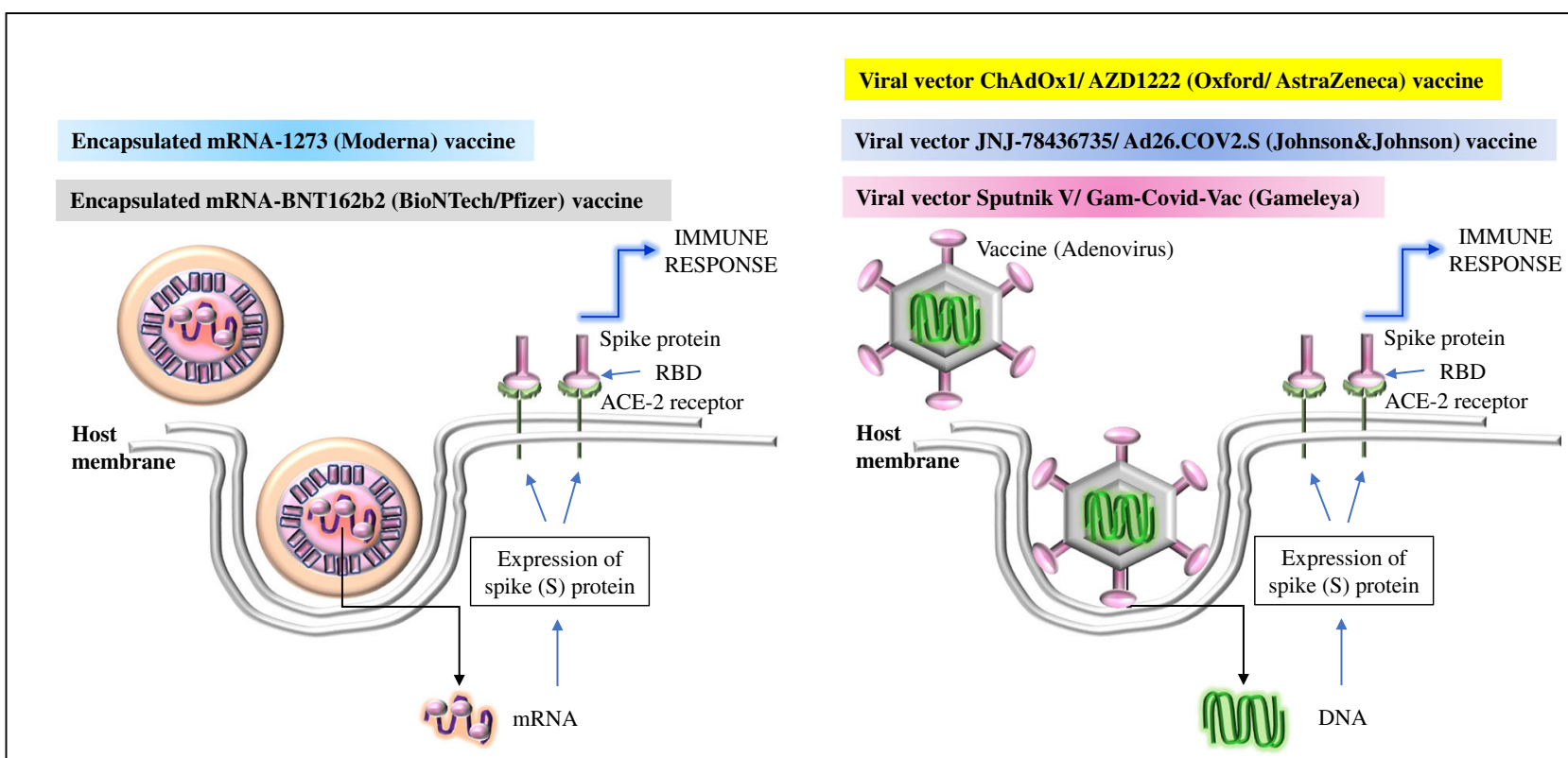

Figure 3 Schematic presentation of the mode of actions of mRNA and viral vector vaccines. The mechanism of the mRNA-1273 (Moderna) mRNA-BNT162b2 (BioNTech/Pfizer) vaccines and the viral vector

vaccines, i.e., ChAdOx1/AZD1222 (Oxford/AstraZeneca), JNJ78436735/Ad26.COV2.S (Johnson \& Johnson), and Sputnik V/GamCovid-Vac (Gamaleya) are shown. Details are given in the text.

of $\mathrm{S}$ protein reactive and the RBD-binding $\mathrm{IgG}$ as well as the required $\mathrm{T}$ cell responses [2]. The viral vector vaccine, ChAdOx1 nCoV-19 (Oxford/AstraZeneca), the doublestranded (ds) DNA encoding the spike protein (kept in a safe virus as shown in Figure 3), has been assessed in four clinical trials across three continents, and the vaccine showed $70.4 \%$ effectiveness after two dose administrations against symptomatic COVID-19 [12]. JNJ-78436735/Ad26.COV2.S (Johnson \& Johnson) also follows the similar physiology as ChAdOx1 nCoV-19 vaccine (Figure 3); and it is a recombinant, replication-incompetent adenovirus serotype 26 (Ad26) vector vaccine which encodes and stabilizes SARS-CoV-2 spike protein [13]. The vaccine has been found to elicit the required immune responses including the spike $(\mathrm{S})$ protein-specific responsiveness of CD4+T cells, CD8+ cells, and the T helper cell 1 (Th1) in more than 60\% cases ( NCT04436276); and the vaccine has been found to be effective up to $72 \%$ [13]. Generation of vigorous antibody responses (neutralizing antibodies inhibiting the virus binding to its receptor) to the spike protein of the SARS-CoV-2 by the administration of the Sputnik V/Gam-Covid-Vac (Gamaleya) is also made of ds DNA encoding the spike protein which has also been observed in the earlier phase clinical trials with proven efficacy $(91 \%)$ of this vaccine expressing the spike protein as shown in Figure 3 [14].

\section{Other Potential COVID-19 Vaccine Candidates}

NVX-CoV2373 (Novavax), a recombinant SARS-CoV-2 nanoparticle vaccine (nanoparticles are coated with synthetic

spike proteins), composed of trimeric full-length spike glycoproteins and Matrix-M1 adjuvant has been found to elicit the IgG anti-spike protein response as well as the secondary T-cell responses [15]. The efficacy has been estimated up to $96 \%$. A scheme of such nanoparticle-based vaccine has been elucidated in Figure 4. BBIBP-CorV (Sinopharm), the chemically inactivated (by employing $\beta$-propiolactone so that the virus cannot replicate although the viral proteins remain intact) SARS-CoV-2 vaccine has been reported to trigger the production of neutralizing antibodies as well with $79 \%$ efficacy [16]. Like BBIBP-CorV, the Corona Vac (developed by Sinovac) is another inactivated virus platform vaccine (employing $\beta$ propiolactone) which targets the spike protein, thereby hindering the viral entry into the host cells with a 50\% efficacy [17•]. On the contrary, the BBV152/Covaxin, using the same platform, has been noticed with $81 \%$ efficacy in terms of immunogenicity $[17 \bullet]$.

\section{Mutations Within SARS-CoV-2 Spike (S) Protein and the Effectiveness of the COVID-19 Vaccines}

Proper vaccination is expected to stimulate immune responses which may neutralize SARS-CoV-2. However, the enduring supervision has unraveled the occurrence of variants resulting from the mutations in the viral spike (S) protein which serves as the principal target of neutralizing antibodies $[2,6,18,19]$. This is to be noted that even a small number of mutations may 
Figure 4 Mode of action of the nanoparticle vaccine NVXCoV2373 (Novavax). Details are given in the text.

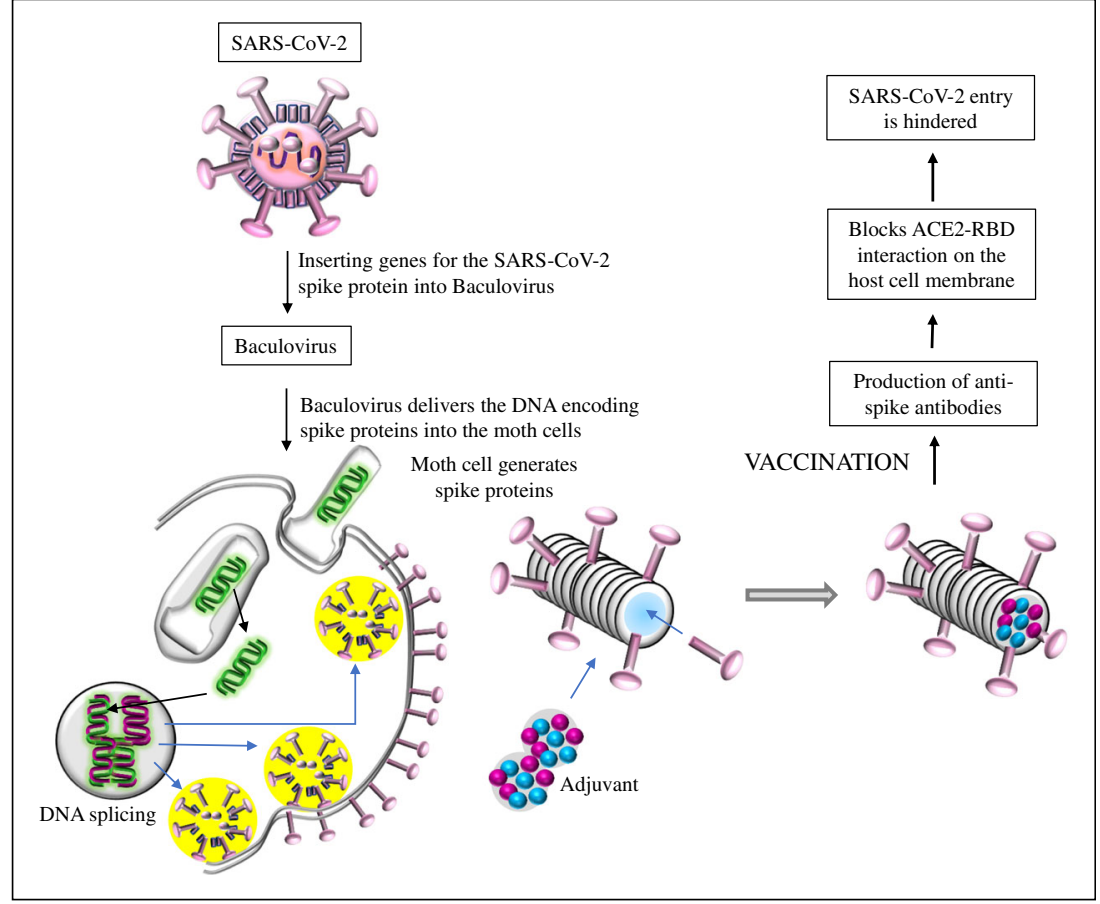

render the virus avoid the host protective immunity meaning that the vaccines are ineffective [18].

Indeed, the genetic reassortment and drifting are not uncommon in the influenza viruses [1]. Similarly, in SARSCoV-2, such genomic changes have been identified as well $[17,18]$. Moreover, it is really arduous to determine the safety and efficacy of the ongoing under-trial vaccines until any vaccine shows the full clinical effectiveness (in terms of antibody production, T-cell response, and the elicitation of cellmediated immunity) in phase III considering the vaccine primary and secondary dosage, vaccine storage conditions, as well as the underlying conditions of the individuals from a large population [17•]. In addition, the SARS-CoV-2 mutation with a varying changeable epitope may take place which may render the existing vaccines incompatible against SARSCoV-2 due to the amino acid changes in the ACE2-RBD receptor [17, 23-25].

The study conducted by Garcia-Beltran et al. (2021) revealed that 5 strains with the RBD mutations (K417N/T, E484K, and N501Y) were extremely resistant to neutralization by the BNT162b2 and mRNA-1273 vaccines [18]. The adenovirus-based vaccines AZD1222 (AstraZeneca) and JNJ78436735 (Johnson \& Johnson), the nanoparticle-based vaccine NVX-CoV2373 (Novavax), and the inactivated protein vaccine Cor also revealed the decreased efficacy due to mutations within the viral spike $(\mathrm{S})$ protein [18]. Considering such mutations and the therapeutic possible vaccine-resistant SARS-CoV-2 variants, Kruse (2020) proposed the ACE2-Fc therapy which is capable to repel the viral entry using a soluble version of the viral ACE2 receptor fused to an immunoglobulin Fc domain (ACE2-Fc) [30]. Such therapy is expected (1) to deliver a neutralizing antibody which can avoid any viral escape with the concomitant recruitment of the host protective immune cells for long term, (2) to augment the decreased ACE2 levels along the lungs during SARS-CoV-2 spread, and (3) thus to directly mitigate the acute respiratory distress syndrome (ARDS) [30].

\section{Conclusion}

Although the results of the randomized clinical trials of the currently used vaccines as well as some repurposed drugs seem to be operative among the COVID-19 patients, researchers require to employ continuous surveillance to observe the long-term immunity as well as the safety concerns. Because of the growing variants of SARS-CoV-2, substituting the existing vaccine's spike $(\mathrm{S})$ protein with the redecorated molecule for achieving the necessary changes in specific amino acid or by inserting newfangled molecule(s) to the prevailing vaccine formulation may be an effective strategy to maintain the sustainability of the vaccines' potential against the new SARS-CoV-2 strains. Constant genomic analysis together with watching the host protective immunity against the new variety of the vaccine(s) against the emerging variants may improve designing new vaccines with required modifications, i.e., swapping the currently used vaccine's spike protein with a remodeled molecule consisting of new amino acid or other novel molecule(s) resulting in a multivalent vaccine may be operative against the new variants of SARS-CoV-2. 
Code Availability Not applicable.

Author Contribution Conceptualization: RN. Literature survey: RN. Formal analysis: RN. Investigation: RN. Resources: RN. Writingoriginal draft preparation: RN. Editing: RN. Visualization: RN. Supervision: RN.

Data Availability Not applicable.

\section{Compliance with Ethical Standards}

Consent to Participate (Ethics) Not applicable.

Consent to Publish (Ethics) The author read and approved the final manuscript for publication.

Conflict of Interest The author declares no competing interests.

Animal Research (Ethics) Not applicable.

Human and Animal Rights and Informed Consent Not applicable.

\section{References}

Papers of particular interest, published recently, have been highlighted as:

- Of importance

•- Of major importance

1. Noor R, Maniha SM. A brief outline of respiratory viral disease outbreaks: 1889 - till date on the public health perspectives. Virus Dis. 2020;31:441-9. https://doi.org/10.1007/s13337-020-00628-5.

2. Noor R. Developmental status of the potential vaccines for the mitigation of the COVID-19 pandemic and a focus on the effectiveness of the Pfizer-BioNTech and Moderna mRNA vaccines. Curr Clin Microbiol Rep. 2021. https://doi.org/10.1007/s40588-02100162-y.

$3 . \bullet$ Noor R. A comparative review of pathogenesis and host innate immunity evasion strategies among the severe acute respiratory syndrome coronavirus 2 (SARS-CoV-2), severe acute respiratory syndrome coronavirus (SARS-CoV) and the Middle East respiratory syndrome coronavirus (MERS-CoV). Arch Microbiol. 2021. https://doi.org/10.1007/s00203-021-02265-y This study provides a thorough description of the molecular aspects of pathogenesis of SARS-CoV-2, SARS-CoV and MERS-CoV. Since the viral entry is the first step of pathogenesis, a distinctive explanation about the viral spike protein-host receptors is given.

4. Asaduzzaman SAI, Zakaria A, Kheya IS, Fahad N, Sikandar YB, Noor R. A comparative study between the severe acute respiratory syndrome-coronavirus-2, severe acute respiratory syndrome coronavirus, and the Middle East respiratory syndrome coronavirus. Biomed Biotechnol Res J. 2020;4:S65-74. https://doi.org/10. 4103/bbrj.bbrj_99_20.

5. Wrapp D, Wang N, Corbett KS, Goldsmith JA, Hsieh CL, Abiona O, et al. Cryo-EM structure of the 2019-nCoV spike in the prefusion conformation. Science. 2020;367(6483):1260-3. https:// doi.org/10.1126/science.abb2507.

6. Noor R. Antiviral drugs against severe acute respiratory syndrome coronavirus 2 infection triggering the coronavirus disease-19 pandemic. Tzu Chi Med J. 2020;33:7-12. https://doi.org/10.4103/ tcmj.tcmj 10020.

7. Sikandar YB, Shabnam I, Noor R. Remdesivir and dexamethasone: the two eligible candidate drugs against severe acute respiratory syndrome coronavirus 2 (SARS-CoV-2) infection. Biomed Res J. 2020. https://doi.org/10.4103/BMRJ.BMRJ_10_20.

8. WHO (World Health Organization) Coronavirus diseases (COVID19) dashboard. Updated on 5:46pm CEST, 4 June 2021. https:// covid19.who.int/ Accessed on June 52021.

9. Kaur SP, Gupta V. COVID-19 vaccine: a comprehensive status report. Virus Res. 2020;288:198114. https://doi.org/10.1016/j. virusres.2020.198114.

10. Baden LR, El Sahly HM, Essink B, Kotloff K, Frey S, Novak R, et al. Efficacy and safety of the mRNA-1273 SARS-CoV-2 vaccine. N Engl J Med. 2021;384(5):403-16. https://doi.org/10.1056/ NEJMoa2035389.

11. Chagla Z. The BNT162b2 (BioNTech/Pfizer) vaccine had 95\% efficacy against COVID-19 $\geq 7$ days after the 2 nd dose. Ann Intern Med. 2021;174(2):JC15. https://doi.org/10.7326/ ACPJ202102160-015.

12. Voysey M, Clemens SAC, Madhi SA, Weckx LY, Folegatti PM, Aley PK, et al. Safety and efficacy of the ChAdOx1 nCoV-19 vaccine (AZD1222) against SARS-CoV-2: an interim analysis of four randomised controlled trials in Brazil, South Africa, and the UK. Lancet. 2021;397(10269):99-111. https://doi.org/10.1016/ S0140-6736(20)32661-1.

13. Sadoff J, Le Gars M, Shukarev G, Heerwegh D, Truyers C, de Groot AM, et al. Interim results of a phase 1-2a trial of Ad26.COV2.S Covid-19 Vaccine. N Engl J Med. 2021: oa2034201. https://doi.org/10.1056/NEJMoa2034201.

14. Jones I, Roy P. Sputnik V COVID-19 vaccine candidate appears safe and effective. Lancet. 2021;397(10275):642-3. https://doi.org/ 10.1016/S0140-6736(21)00191-4.

15. Keech C, Albert G, Cho I, Robertson A, Reed P, Neal S, et al. Phase 1-2 trial of a SARS-CoV-2 recombinant spike protein nanoparticle vaccine. N Engl J Med. 2020;383(24):2320-32. https://doi.org/10. 1056/NEJMoa2026920.

16. Wang H, Zhang Y, Huang B, Deng W, Quan Y, Wang W, et al Development of an inactivated vaccine candidate, BBIBP-CorV, with potent protection against SARS-CoV-2. Cell. 2020;182(3): 713-721.e9. https://doi.org/10.1016/j.cell.2020.06.008.

17. Dutta AK. Vaccine against Covid-19 disease - present status of development. Indian J Pediatr. 2020;87(10):810-6. https://doi.org/ 10.1007/s12098-020-03475-w This study provides the insight of the current stages of development of the novel COVID-19 vaccines together with the challenges that may be encountered.

18. Garcia-Beltran WF, Lam EC, Denis KS, Nitido AD, Garcia ZH, Hauser BM, et al. Circulating SARS-CoV-2 variants escape neutralization by vaccine-induced humoral immunity. medRxiv. 2021 https://doi.org/10.1101/2021.02.14.21251704.

19. Dos Santos WG. Impact of virus genetic variability and host immunity for the success of COVID-19 vaccines. Biomed Pharmacother. 2021;136:111272. https://doi.org/10.1016/j.biopha.2021.111272.

20. Jomah S, Asdaq SMB, Al-Yamani MJ. Clinical efficacy of antivirals against novel coronavirus (COVID-19): a review. J Infect Public Health. 2020;13(9):1187-95. https://doi.org/10.1016/j.jiph. 2020.07.013.

21. Zhong H, Wang Y, Zhang ZL, Liu YX, Le KJ, Cui M. Efficacy and safety of current therapeutic options for COVID-19 - lessons to be learnt from SARS and MERS epidemic: a systematic review and meta-analysis. Pharmacol Res. 2020;157:104872. https://doi.org/ 10.1016/j.phrs.2020.104872.

22. Lian N, Xie H, Lin S, Huang J, Zhao J, Lin Q. Umifenovir treatment is not associated with improved outcomes in patients with coronavirus disease 2019: a retrospective study. Clin Microbiol Infect. 2020;26:917-21. https://doi.org/10.1016/j.cmi.2020.04.026. 
23. Awadasseid A, Wu Y, Tanaka Y, Zhang W. SARS-CoV-2 variants evolved during the early stage of the pandemic and effects of mutations on adaptation in Wuhan populations. Int $\mathrm{J}$ Biol Sci. 2021;17(1):97-106. https://doi.org/10.7150/ijbs.47827.

24.• Dos Santos WG. Impact of virus genetic variability and host immunity for the success of COVID-19 vaccines. Biomed Pharmacother. 2021;136:111272. https://doi.org/10.1016/j.biopha.2021.111272 This study provides the depiction on the possible reinfection of the recovered individuals from COVID-19; hence a doubtful duration of the immunity; and discussed the impact of SARS-CoV-2 mutations which may render the currently used vaccines questionable regarding their safety and efficacy.

25. Tang X, Wu C, Li X, Song Y, Yao X, Wu X, et al. On the origin and continuing evolution of SARS-CoV-2. Natl Sci Rev. 2020: nwaa036. https://doi.org/10.1093/nsr/nwaa036.

26. Forster P, Forster L, Renfrew C, Forster M. Phylogenetic network analysis of SARS-CoV-2 genomes. Proc Natl Acad Sci U S A. 2020;117(17):9241-3. https://doi.org/10.1073/pnas.2004999117.

27. Korber B. Tracking changes in SARS-CoV-2 spike: evidence that D614G increases infectivity of the COVID-19 virus. Cell. 2020;182(4):812-27. https://doi.org/10.1016/j.cell.2020.06.043.

28. Promi AT, Bristi SI, Akhter F, Noor R. Severe acute respiratory syndrome coronavirus (SARS-CoV-2): why is it so lethal? MOJ Biol Med. 2021;6(2):65-7. https://doi.org/10.15406/mojbm.2021. 06.00131.

29. Prajapat M, Sarma P, Shekhar N, Avti P, Sinha S, Kaur H, et al. Drug targets for corona virus: a systematic review. Indian J Pharm. 2020;52:56-65.
30. Kruse RL. Therapeutic strategies in an outbreak scenario to treat the novel coronavirus originating in Wuhan, China. F1000Res 2020; 9:72.

31. De A, Tiwari A, Dash M, Sinha A. ACE2 mutation might explain lower COVID-19 burden in malaria endemic areas. Hum Cell. 2021;34(2):702-5. https://doi.org/10.1007/s13577-021-00489-0.

32. Wu J, Deng W, Li S, Yang X. Advances in research on ACE2 as a receptor for 2019-nCoV. Cell Mol Life Sci. 2021 Jan;78(2):53144. https://doi.org/10.1007/s00018-020-03611-x.

33. Li R, Qiao S, Zhang G. Analysis of angiotensin-converting enzyme 2 (ACE2) from different species sheds some light on cross-species receptor usage of a novel coronavirus 2019-nCoV. J Inf Secur. 2020;80(4):469-96. https://doi.org/10.1016/j.jinf.2020.02.013.

34. Gómez CE, Perdiguero B, Esteban M. Emerging SARS-CoV-2 variants and impact in global vaccination programs against SARS-CoV-2/COVID-19. Vaccines (Basel). 2021;9(3):243. https://doi.org/10.3390/vaccines9030243.

35. Heinz FX, Stiasny K. Profiles of current COVID-19 vaccines. Wien Klin Wochenschr. 2021;133(7-8):271-83. https://doi.org/10.1007/ s00508-021-01835-w.

36. Huang Y, Yang C, Xu X-f, Xu W, Shu-wen L. Structural and functional properties of SARS-CoV-2 spike protein: potential antivirus drug development for COVID-19. Acta Pharmacol Sin. 2020;41:1141-9. https://doi.org/10.1038/s41401-020-0485-4.

Publisher's Note Springer Nature remains neutral with regard to jurisdictional claims in published maps and institutional affiliations. 